\title{
Viabilidad de Ovocitos Vitrificados y Madurados in Vitro de Gata Doméstica Adulta (Felis catus) en Estación Reproductiva
}

\author{
Vitrified and in Vitro Matured Oocytes Viability from \\ Adult Housecat (Felis catus) in Reproductive Season
}

Juan J. Calvo*; Deborah Robert*; Mónica Viqueira* \& Paula Lombide*

CALVO, J. J.; ROBERT, D.; VIQUEIRA, M. \& LOMBIDE, P. Viabilidad de ovocitos vitrificados y madurados in vitro de gata doméstica adulta (Felis catus) en estación reproductiva. Int. J. Morphol., 33(4):1242-1247, 2015.

RESUMEN: La vitrificación de ovocitos de mamíferos es considerada una técnica en experimentación. La supervivencia de los ovocitos es extremadamente variable, según las técnicas utilizadas e influida por una serie de condiciones. El objetivo de éste trabajo fue determinar los efectos de la vitrificación en ovocitos de gata domestica adulta en estación reproductiva y madurados in vitro. Se obtuvieron 33 ovarios correspondientes a hembras de más de un año en buen estado nutricional y sin tratamiento hormonal, ovariectomizadas en domicilio del propietario por profesional veterinario. Los ovarios fueron trasladados al laboratorio y se fragmentaron por microdisección bajo microscopio estereoscópico en caja de Petri con solución de buffer fosfato salino modificado con suero de ternero inactivado y antibióticos para la obtención, evaluación y selección de los complejos cúmulo-ovocitos (CCOs) de buena calidad. Se vitrificaron 506 CCOs en pajuelas de $0.25 \mathrm{ml}$ con $10-12$ ovocitos cada una y almacenados por 30 días en nitrógeno líquido (N2) a -196 ${ }^{\circ} \mathrm{C}$. Posteriormente se desvitrificaron las pajuelas, recuperándose $320 \mathrm{CCOs}$, los que fueron madurados in vitro. Transcurrido ese tiempo se evaluaron los CCOs, descartándose 50 por presentar signos de degeneración. En los 270 restantes se observó buena expansión del cúmulo, citoplasma uniforme y oscuro e integridad de la zona pelúcida. A estos últimos se los dividió en dos grupos similares para evaluar la viabilidad, a uno se lo coloreó con metil tiazol tetrazolio y al otro con Azul Tripán. En ambos se constató un resultado positivo para la viabilidad de los CCOs. El análisis de los resultados nos permite concluir que la vitrificación comprometió la integridad de un importante número de CCOs aunque más del 50\% respondió en cultivo favorablemente, mostrando signos de viabilidad esperados. Estos resultados hacen necesarios la profundización en el mejoramiento del protocolo para incrementar el porcentaje de viabilidad.

PALABRAS CLAVE: Ovocitos; Vitrificación; Maduración; Gata; Viabilidad.

\section{INTRODUCCIÓN}

La biodiversidad ha descendido considerablemente en el planeta y América Latina no escapa a esta tendencia mundial. Vivimos en una de las regiones privilegiadas por su riqueza de fauna y flora y tenemos la responsabilidad de buscar las mejores estrategias para su preservación. A veces la preservación del medio natural no es posible debido a su grado de deterioro por el desarrollo urbano, agrícola, ganadero, forestal o industrial; existiendo situaciones en las que la disminución o desaparición de una especie no está asociada a una manifiesta reducción de su hábitat natural, sino a la falta de alimentación, exceso de caza o problemas de carácter sanitario (Roldán \& Garde, 2004). Existen en la actualidad una variedad de biotecnologías reproductivas (fertilización in vitro, transferencia de embriones) que pueden aplicarse en programas de conservación de especies en peligro de extinción. Para la utilización de estas tecnologías es necesario conocer en profundidad la fisiología de la re- producción de los animales silvestres, si bien en este sentido se han obtenido importantes avances, persisten limitantes para una mayor eficiencia en gatos y felinos salvajes (Franzosi Mattos et al., 2003). La conservación por frío de células humanas es una tecnología ampliamente utilizada en medicina y en el campo de la reproducción la congelación de espermatozoides tiene más de 50 años a nivel mundial. En humanos la congelación de ovocitos busca resolver a nivel médico, ético, legal y práctico la problemática de la reproducción asistida (Marina et al., 2002). Desde hace varios años se disponen de Banco de Embriones y Banco de Semen y recientemente se están incorporando los ovocitos a Banco de Gametos de humanos y de varias especies animales. Hay dos estrategias para criopreservar: la criopreservación lenta, en que la temperatura disminuye lentamente, comúnmente a una tasa de $-0,3{ }^{\circ} \mathrm{C} / \mathrm{min}$; y la vitrificación, un tipo de crio-preservación ultrarrápido, con 
inmersión directa en nitrógeno líquido (Kim et al., 2010). Las sustancias crioprotectoras pueden clasificarse como penetrantes cuando ingresan a la célula como el glicerol, etilenglicol, dimetilsulfoxido (DMSO), propanodiol (PROH) o no penetrantes como la sucrosa y glucosa que por tratarse de moléculas grandes no atraviesan el ovolema. La criopreservación exitosa de gametos femeninos habría permitido superar las diferencias de tiempo y espacio que a menudo son un obstáculo para la investigación y trabajo de campo de especies de felinos. El gato doméstico (Felis catus) es el único de las 37 especies de la familia felidae que no vive amenazado o en peligro de extinción. Proviene aparentemente, de la cruza del gato montés europeo (Felis silvestris) y del gato doméstico africano (Felis lybica) en Europa y Asia. Su domesticación habría comenzado en el antiguo Egipto, alrededor del año 2.000 A.C. Los romanos habrían introducido esta especie a Bretaña alrededor del año 300 D.C. Luego, serían los colonizadores europeos los encargados de introducir al gato doméstico en el resto del mundo (Serpell, 1988). Además de mascota, en los últimos tiempos también este ha sido utilizado como modelo biomédico para estudios en la fisiología y biotecnologías en programas de reproducción asistida de pequeños y grandes felinos silvestres (Jewgenow \& Paris, 2006; Pope, 2000). La vitrificación de ovocitos de mamíferos es considerada una técnica en experimentación. La supervivencia de los ovocitos es extremadamente variable, según las técnicas utilizadas e influida por una serie de condiciones. La criopreservación de ovocitos se ha logrado con diversos grados de éxito en las pocas especies en que ha sido ampliamente estudiado, incluyendo el gato doméstico. Aunque han nacido gatitos después de una tasa de criopreservación controlada y transferencia de embriones derivados in vitro e in vivo, la criopreservación de ovocitos de gato sigue siendo un área urgente de mayor exploración (Cocchia et al., 2010; Pope et al., 2012) ya que todavía no se considera una herramienta confiable y repetible (Apparicio et al., 2013; Mikolajewska et al., 2012).

El estudio de la viabilidad y la proliferación celular es un factor importante para evaluar las respuestas de una o varias poblaciones celulares a diferentes factores. La reducción de las sales de tetrazolio (MTT) por parte de las enzimas mitocondriales ha sido utilizada en ensayos de cuantificación de células viables (Calvo et al., 2004). Así mismo la penetración del Azul Tripán en el citoplasma de las células con membrana plasmática alterada se usa comúnmente en microscopía para diferenciar las células vivas de las muertas, también llamado método de tinción por exclusión porque el colorante no ingresa en las células vivas sino en las muertas (Filipiak \& Larocca, 2012). El objetivo del presente trabajo fue determinar la viabilidad de ovocitos vitrificados y madurados in vitro de gata doméstica adulta en estación reproductiva.

\section{MATERIAL Y MÉTODO}

Obtención de muestra. Los ovarios de 17 gatas adultas en buen estado nutricional y sin tratamientos hormonales fueron obtenidos por ovariectomía, en domicilio del propietario por profesional veterinario. Posteriormente fueron transportados al laboratorio en solución salina fisiológica $(\mathrm{NaCl}$ $0,9 \%)$ con gentamicina $(100 \mu \mathrm{g} / \mathrm{ml})$ y refrigerados a $4{ }^{\circ} \mathrm{C}$. dentro de las dos horas posterior a su extracción.

Los ovarios se fragmentaron por microdisección, con tijera y escalpelo, en caja de Petri de 90 mm con solución de tampón fosfato salino modificado por Dulbecco (m-PBS, Gibco Laboratories, Grand Island, NY, USA) suplementado con suero de ternero inactivado (CS, Gibco Laboratories, Grand Island, NY, USA) y antibióticos (100.000 UI/ml de penicilina y $10 \mathrm{mg} / \mathrm{l}$ de estreptomicina, ATB, Sigma Chemical Co. St. Louis, MO, USA). Los complejos cúmulo-ovocitos (CCOs) liberados fueron colectados bajo microscopio estereoscópico (Nikon, Modelo SMZ-2T, Japan) con pipetas Pasteur estériles y transferidos a caja de Petri de 35 mm con igual solución utilizada durante la microdisección. Los CCOs obtenidos fueron clasificados en cuatro categorías (Wood \& Wildt 1997). Solo los CCOs de grado A y B fueron utilizados (3 o más capas de células de la granulosa, integridad de la zona pelúcida y citoplasma homogéneo y oscuro) para la vitrificación y maduración in vitro (Fig. 1).

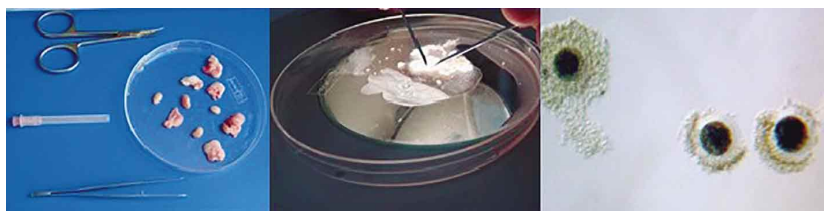

Fig. 1. Obtención y clasificación de cúmulos-ovocitos (CCOs).

Vitrificación. Según este protocolo los ovocitos fueron lavados en una solución de TCM-199, CS, ATB, y expuestos gradualmente a una solución de equilibrio de TCM-199, CS, ATB y etilenglicol 1,5 M como crioprotector penetrante en PBS. A continuación se sometieron a una rápida exposición a la solución de vitrificación con CS, ATB, y etilenglicol suplementada con sucrosa $0,1 \mathrm{M}$ como crioprotector no penetrante. Los CCOs vitrificados fueron incluidos en pajuelas de 0,25 ml en sistema de columnas con solución diluyente de TCM-199, CS, vitelo y sucrosa. Se almacenaron un total de cuarenta y seis pajuelas con 10-12 CCOs cada una por treinta días en nitrógeno líquido $\left(\mathrm{N}_{2}\right)$ a $-196{ }^{\circ} \mathrm{C}$ (Fig. 2) (Kuwayama et al., 1997).

Desvitrificación. Siete pajuelas se perdieron por defectos de sellado y/o manipulación. Las treinta y nueve restantes 


\begin{tabular}{|c|c|c|c|c|c|c|}
\hline & $\begin{array}{l}\text { TCM } \\
-199\end{array}$ & & ATB & VTELO & SUCROSA & ERLENGLICOL \\
\hline $\begin{array}{l}\text { Lavado } \\
\text { (W) }\end{array}$ & $\checkmark$ & $\checkmark$ & $\checkmark$ & & & \\
\hline $\begin{array}{l}\text { Diluyento } \\
\text { (D) }\end{array}$ & $\checkmark$ & $\checkmark$ & & $\checkmark$ & $\checkmark$ & \\
\hline $\begin{array}{l}\text { Equilibramlento } \\
\text { (E) }\end{array}$ & $\checkmark$ & $\checkmark$ & $\checkmark$ & & & $\checkmark$ \\
\hline $\begin{array}{l}\text { Vitrificación } \\
(\mathrm{M})\end{array}$ & & $\checkmark$ & $\checkmark$ & & $\checkmark$ & $\checkmark$ \\
\hline
\end{tabular}
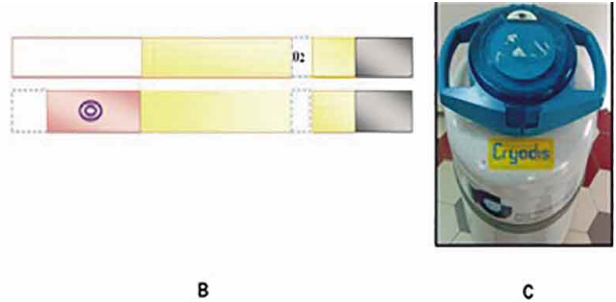

Fig. 2. A) Esquema de los componentes utilizados en las soluciones de vitrificación. B) Almacenamiento en pajuelas de 0,25 ml en N2. C) Tanque de N2.

se desvitrificaron trasladándolas individualmente desde el recipiente de almacenamiento con nitrógeno líquido a un recipiente con agua a $20{ }^{\circ} \mathrm{C}$ por $20 \mathrm{~s}$. A continuación se retiraron y secaron con papel absorbente. Finalmente se cortaron con tijera sus extremos descargando su contenido en caja de Petri (Fig. 3).

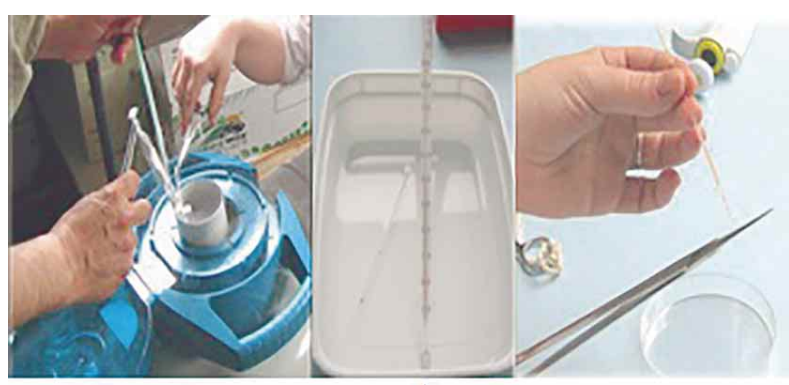

A

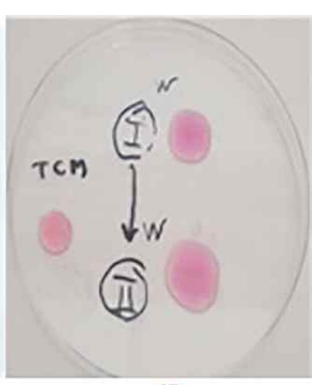

B
Fig. 3. Desvitrificación de pajuelas. B) Lavado de los CCOs.

Maduración in vitro. Los CCOs recuperados fueron madurados in vitro (MIV) en gotas $(50 \mu \mathrm{l})$ de medio de cultivo TCM 199 con 10\% de CS y ATB, cubiertas con aceite mineral estéril, a $38^{\circ} \mathrm{C}$, con $99 \%$ de humedad relativa y atmósfera de anhídrido carbónico $\left(\mathrm{CO}_{2}\right)$ al $5 \%$ por $48 \mathrm{~h}$. Transcurrido ese tiempo, se descartaron cincuenta CCOs con signos de degeneración. En los CCOs restantes se observó buena expansión del cúmulo, citoplasma uniforme y oscuro e integridad de la zona pelúcida (Fig. 4).

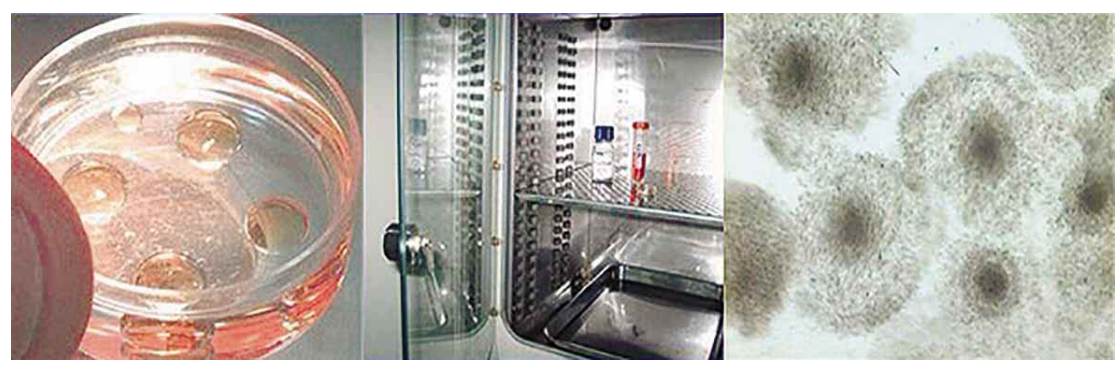

Fig. 4. Maduración in vitro de los CCOs.

Ensayos de Viabilidad. Para determinar la viabilidad de los CCOs recuperados y madurados in vitro se utilizaron dos procedimientos de evaluación dividiendo los CCOs en dos grupos de características similares, en uno se utilizó MTT y en el otro Azul Tripán.

Incubación con MTT. Un grupo de 140 CCOs fueron incubados según el méto- do descrito previamente basado en la reducción metabólica del Bromuro de 3-(4-5-dimetiltiazol-2-ilo)-2,5difeniltetrazol) (Calvo et al.). El MTT es disuelto en m-PBS a una concentración de $5 \mathrm{mg} / \mathrm{ml}$ y posteriormente filtrado (solución madre). Los CCOs se colocaron individualmente mediante pipeta Pasteur en cada cava de una placa de ELISA-96, con $10 \mu \mathrm{l}$ de solución madre de MTT en $100 \mu \mathrm{lde}$ mPBS e incubados durante $4 \mathrm{~h}$ a $37^{\circ} \mathrm{C}$, $5 \% \mathrm{CO}_{2}$ y $99 \%$ de humedad. El MTT al ser reducido por la enzima mitocondrial succinato-deshidrogenasa cambia de incoloro o débilmente amarillento a azul oscuro o purpura debido a la formación de un precipitado de formazan. Después de la incubación, los CCOs fueron observados, evaluados y clasificados. Según la respuesta de los CCOs observada, se clasificaron como positivo en aquellos que presentaron cambio en la coloración y como negativo donde no se manifestó dicho cambio (Fig. 5).

Incubación con Azul Tripán. Fueron incubados 130 CCOs en Azul Tripán para evaluar su viabilidad. El Azul Tripán, es un colorante azoico que se utiliza para ensayos de viabilidad que permiten diferenciar células vivas de células muertas. El Azul Tripán al 0,4\% (Sigma Chemical Co. St. Louis, MO, USA) fue disuelto en agua destilada y filtrado con filtro $0,22 \mu \mathrm{m}$ para evitar agentes bacterianos. Los CCOs se colocaron individualmente mediante pipeta Pasteur en cada cava de una placa de ELISA-96, con $100 \mu \mathrm{l}$ de solución de Azul Tripán 0,4\% en $100 \mu 1$ de m-PBS e incubados durante 5 minutos a $37{ }^{\circ} \mathrm{C}, 5 \% \mathrm{CO}_{2}$ y $99 \%$ de humedad. Es una molécula coloreada de gran peso molecular que solo es capaz de entrar en las células que tienen la membrana plasmática alterada. Por tanto una célula viva en perfecto estado se observará incolora mientras que una célula muerta o muy alterada se observará azul como se demuestra en la Figura 6. 


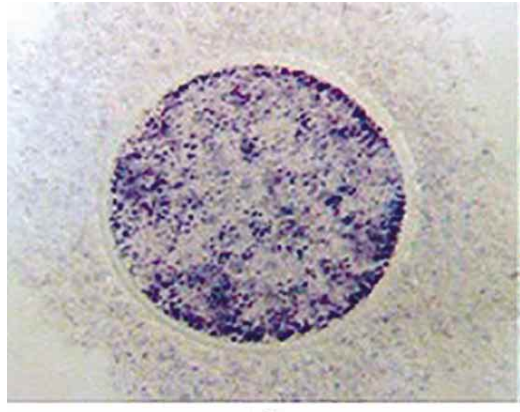

A

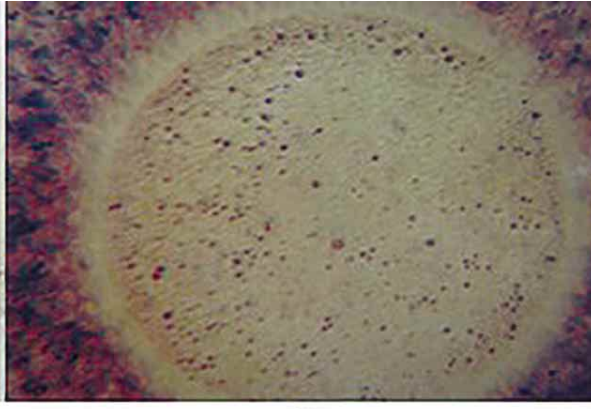

B
Fig. 5. Ensayo de viabilidad con MTT. A) CCOs positivo en donde se observa reducción metabólica del MTT. B) CCOs negativo. Microscopía de luz 40X.

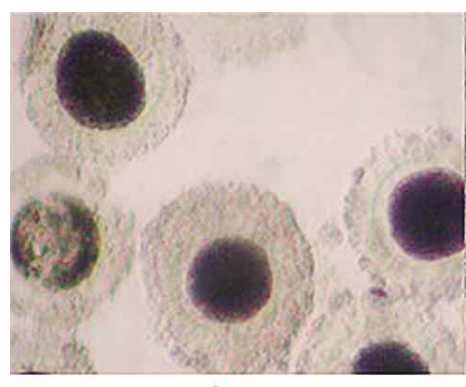

A

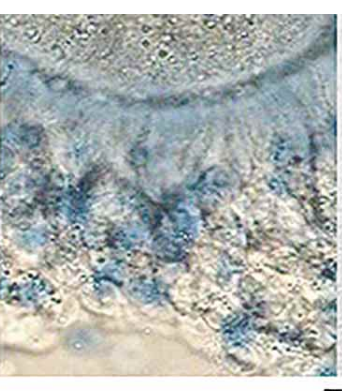

B
Fig. 6. Ensayo de viabilidad con Azul Tripán. A) CCOs viables incoloros. Microscopía de luz 20X. B) CCOs no viables azules. Microscopía de luz 100X y 20X, respectivamente.

\section{RESULTADOS}

En el presente estudio fueron utilizados treinta y tres ovarios, en fase folicular, de gata adulta doméstica en estación reproductiva. Un total de quinientos ocho CCOs de grados A y B fueron recuperados mediante la microdisección. El número promedio de CCOs obtenido por ovario fue de $15,39 \pm 0,84$. Se evaluaron un total de 270 CCOs para ambos ensayos de viabilidad. Los resultados se expresan en la Tabla I.

\section{DISCUSIÓN}

El ovocito es la célula aislada más grande del organismo y sensiblemente esférica cuya relación superficie y volumen es de aproximadamente tres cuartos del radio, es decir que a mayo radio la relación de superficie de membrana respecto a su volumen es menor. La célula con mayor volumen contiene más agua y proporcionalmente menos superficie de membrana para la salida del agua y la entrada de sustancia crioprotectoras penetrantes. Estas características son de relevancia para el proceso de enfriamiento y calentamiento al que son sometidas en el proceso de vitrificación.

Los eventos ocurridos durante los procesos de vitrificación, desvitrificación y maduración de los CCOs influyen significativamente en su capacidad de viabilidad. La vitrificación es una técnica de criopreservación valida con la que hay todavía una corta experiencia. Si bien la vitrificación puede ayudar a criopreservar células muy sensibles a bajas temperaturas (Mazur et al., 1992) el descenso térmico es muy rápido y el agua intracelular no tiene tiempo para salir. Los solutos y el agua de la célula pasan de un estado líquido a sólido "vítreo", la célula no se deshidrata. Para cada tipo celular hay un ritmo óptimo de enfriamiento. Este ritmo condiciona el proceso de desvitrificación. El ritmo de desvitrificación es también crítico y rápido y el problema que puede suceder es la recristalización con formación de cristales de hielo intracelular que dañen por ejemplo a los ovocitos. $\mathrm{Si}$ la desvitrificación es lenta, al introducir el ovocito al nitrógeno líquido ($196^{\circ} \mathrm{C}$ ), los pequeños cristales de hielo actuarán como núcleos de cristalización, crecerán en tamaño y dañarán al ovocito. Durante el proceso de vitrificación los CCOs sufren procesos de daño morfológico y funcional que son críticos para su posterior desarrollo (Apparicio et al.). La maduración in vitro de la totalidad de los CCOs en diferentes medios de cultivo

Tabla I. Número de CCOs obtenidos por microdisección, vitrificados, madurados y evaluados su viabilidad con los métodos de MTT y Azul Tripán.

\begin{tabular}{lccccccc}
\hline CCOs & $\begin{array}{c}\text { Obtenidos y } \\
\text { Vitrificados }\end{array}$ & $\begin{array}{c}\text { Madurados } \\
\text { in vitro }\end{array}$ & Descartados & $\begin{array}{c}\text { Totales } \\
\text { evaluados } \\
\text { MTT }\end{array}$ & $\begin{array}{c}\text { Viables } \\
\text { (MTT) } \\
\text { n (\%) }\end{array}$ & $\begin{array}{c}\text { Totales } \\
\text { evaluados } \\
\text { Azul Tripán }\end{array}$ & $\begin{array}{c}\text { Viables (Azul } \\
\text { Tripán) } \\
\text { n (\%) }\end{array}$ \\
\hline $\mathbf{n}$ & 508 & 320 & 50 & 140 & $78(56)$ & 130 & $69(53)$ \\
\hline
\end{tabular}


aún no ha sido posible sin embargo existen una alta correlación entre la calidad de los CCOs y habilidad en la maduración de su núcleo así como de la distribución de los organelos en el citoplasma (Damiani et al., 1995; Leibfried \& First, 1979). Además de la integridad del ovocito resulta de gran importancia la presencia de las células del cúmulo, las que establecen una verdadera cooperación metabólica posibilitando el pasaje de nutriente hacia el ovocito. Según los datos obtenidos en nuestro experimento con la coloración de MTT se demuestra que los CCOs de coloración positiva están probablemente relacionados con la integridad funcional de las mitocondrias, ya que los mismos han mostrado un cambio de coloración de intensidad diferente como consecuencia de la formación de un precipitado de formazán. Así como los datos obtenidos por Azul Tripán que coloreó con diferente grado de intensidad a los ovocitos y células del cúmulo están vinculados a la integridad de la membrana plasmática. El análisis de los resultados nos permite concluir que la vitrificación comprometió la integridad de un importante número de CCOs aunque más del 50\% respon- dió en cultivo favorablemente, mostrando signos de viabilidad esperados. La utilización de los ensayos de viabilidad ratifican los porcentajes reportados previamente (Mikolajewska et al.). Por esto se hace necesaria la profundización en el mejoramiento del protocolo para incrementar el porcentaje de viabilidad. Según los datos obtenidos existe una correlación positiva entre la coloración del MTT y su intensidad con la calidad morfológica de los CCOs (Calvo et al.). La utilización de criterios morfológicos para la evaluación y selección de los CCOs destinados a programas criopreservación han sido utilizados con éxito, sin embargo muchas veces no permiten percibir procesos de atresia o apoptosis incipientes y que configuran anomalías de la cromatina, alteración de la integridad de las mitocondrias, el citoesqueleto o el sistema de membrana. En este sentido se hace necesaria continuar los esfuerzos para lograr las condiciones necesarias que conduzcan a la obtención buenos resultados, contando con probados protocolos de vitrificación de ovocitos y técnicas cuya estandarización determinen aspectos de su funcionalidad o integridad estructural.

CALVO, J. J.; ROBERT, D.; VIQUEIRA, M. \& LOMBIDE, P. Vitrified and in vitro matured oocytes viability from adult housecat (Felis catus) in reproductive season. Int. J. Morphol., 33(4):1242-1247, 2015.

SUMMARY: Vitrification of mammalian oocytes is a technique considered in experimentation. Oocyte survival is extremely variable, according to the techniques used and influenced by a number of conditions. The objective of this study was to determine the effects of adult domestic cat oocyte matured in vitro and vitrified in breeding season. We obtained 33 ovaries from housecats in good nutritional status without hormonal treatment, ovariectomized by a veterinary professional at home. The ovaries were transported to the laboratory and fragmented by microdissection under a stereoscopic microscope in a petri dish with modified buffer saline phosphate solution, inactivated calf serum and antibiotics to the collection, evaluation and selection of good quality cumulus-oocyte complexes (COCs). 506 COCs were vitrified in $0.25 \mathrm{ml}$ straws each with 10-12 oocytes and stored for 30 days in liquid nitrogen $\left(\mathrm{N}_{2}\right)$ at $-196{ }^{\circ} \mathrm{C}$. Then the straws were thawed, recovering $320 \mathrm{CCOs}$, which were matured in vitro. After this time the COCs were evaluated, discarding 50 which showed signs of degeneration. In the remaining 270 COCs good cumulus expansion was observed and also uniformly dark cytoplasm and integrity of the zona pellucida. The latter were divided into two similar groups to assess the feasibility; one was stained with Methylthiazblyl tetrazolium and the other with Trypan Blue. Both tested positive for the viability of the CCOs was found. The analysis of the results allow us to conclude that vitrification compromised the integrity of a large number of CCOs although more than $50 \%$ responded favorably in culture, showing signs of expected viability. According to these results it is necessary to further improve the protocol to increase the percentage of viability.

\section{KEY WORDS: Oocytes; Vitrification; Maturation; Housecat; Viability.}

\section{REFERENCIAS BIBLIOGRÁFICAS}

Apparicio, M.; Ruggeri, E. \& Luvoni, G. C. Vitrification of immature feline oocytes with a commercial kit for bovine embryo vitrification. Reprod. Domest. Anim., 48(2):240-4, 2013.

Calvo, J.; Pérez, V.; Fila, D. \& Campos, E. Evaluación de la viabilidad de ovocitos bovinos seleccionados para maduración y fertilización in vitro mediante la utilización del MTT. Vet. (Montev.), 39(154):7-10, 2004.

Cocchia, N.; Ciani, F.; Russo, M.; El Rass, R.; Rosapane, I.;
Avallone, L.; Tortora, G. \& Lorizio, R. Immature cat oocyte vitrification in open pulled straws (OPSs) using a cryoprotectant mixture. Cryobiology, 60(2):229-34, 2010.

Damiani, P.; Fissore, R. A.; Cibelli, J. B.; Robl, J. M. \& Duby, R.T. Evaluation of cytoplasmic maturation of calf oocytes. Theriogenology, 43(1):191, 1995.

Filipiak, Y. \& Larocca, C. Utilización del azul tripán para diferenciar ovocitos bovinos vivos y muertos en fertilización in vitro. Arch. Zootec., 61(234):309-12, 2012. 
CALVO, J. J.; ROBERT, D.; VIQUEIRA, M. \& LOMBIDE, P. Viabilidad de ovocitos vitrificados y madurados in vitro de gata doméstica adulta (Felis catus) en estación reproductiva. Int. J. Morphol., 33(4):1242-1247, 2015.

Franzosi Mattos, M. R.; Simões-Mattos, L. \& Machado de Silva, L. D. Embryo technology in the domestic cat (Felis catus). Vet. Mex., 34(4):373-88, 2003.

Jewgenow, K. \& Paris, M. C. Preservation of female germ cells from ovaries of cat species. Theriogenology, 66(1):93-100, 2006.

Kim, T. J.; Laufer, L. R. \& Hong, S. W. Vitrification of oocytes produces high pregnancy rates when carried out in fertile women. Fertil. Steril., 93(2):467-74, 2010.

Kuwayama, M.; Hamano, S. \& Nagai, T. Vitrification of bovine blastocysts obtained by in vitro culture of oocytes matured and fertilized in vitro. J. Reprod. Fertil., 96(1):187-93, 1997.

Leibfried, L. \& First, N. L. Characterization of bovine follicular oocytes and their ability to mature in vitro. J. Anim. Sci., 48(1):76-86, 1979.

Marina, S.; Marina, F.; Torres, P. J.; Fosas, N.; Martín, P.; Alcolea, R.; Pérez, N.; Fernández, S.; Arnedo, N.; Jové, I.; Hochman, M. \& Suñol, J. Congelación de ovocitos para reproducción asistida. Rev. Iberoam. Fertil., 19(1):59-68, 2002.

Mazur, P.; Schneider, U. \& Mahowald, A. P. Characteristics and kinetics of subzero chilling injury in Drosophila embryos. Cryobiology, 29(1):39-68, 1992.

Mikolajewska, N.; Müller, K.; Nizanski, W. \& Jewgenowm, K. Vitrification of domestic cat oocytes--effect on viability and integrity of subcellular structures. Reprod. Domest. Anim., 47 (Suppl. 6):295-9, 2012.

Pope, C. E. Embryo technology in conservation efforts for endangered felids. Theriogenology, 53(1):163-74, 2000.

Pope, C. E.; Gómez, M. C.; Kagawa, N.; Kuwayama, M.; Leibo, S. P. \& Dresser, B. L. In vivo survival of domestic cat oocytes after vitrification, intracytoplasmic sperm injection and embryo transfer. Theriogenology, 77(3):531-8, 2012.

Roldán, E. \& Garde, J. Biotecnología de la reproducción y conservación de especies en peligro de extinción. Madrid, Museo Nacional de Ciencias Naturales, 2004. pp.307-38.

Serpell, S. A. The domestication of the cat. In: Turner, D. C. \& Bateson, P. (Eds). The domestic cat: The biology of its behaviour. Cambridge, Cambridge University Press, 1988. pp.151-8.

Wood, T. C. \& Wildt, D. E. Effect of the quality of the cumulusoocyte complex in the domestic cat on the ability of oocytes to mature, fertilize and develop into blastocysts in vitro. J. Reprod. Fertil., 110(2):355-60, 1997.

\author{
Dirección para Correspondencia: \\ Juan José Calvo Marín \\ Laboratorio de Biotecnología de la Reproducción \\ Área Histología y Embriología \\ Facultad de Veterinaria \\ Universidad de la República \\ Alberto Lasplaces 1620. CP 11600 \\ Montevideo \\ URUGUAY
}

Email: juancalvouy@gmail.com

Recibido: 16-04-2015

Aceptado: 02-09-2015 